\title{
VARIABLES QUE INFLUYEN EN LA DESERCIÓN DE ESTUDIANTES UNIVERSITARIOS DE PRIMER AÑO. CONSTRUCCIÓN DE ÍNDICES DE RIESGO DE ABANDONO
}

VARIABLES THAT INFLUENCE FIRST YEAR UNIVERSITY STUDENTS' DROPOUT RATES.CONSTRUCTION OF DROPOUT RISK INDEXES

\author{
Volumen 11, Número 2
}

pp. 1-30

Este número se publicó el 30 de agosto de 2011

\author{
Aldo Merlino \\ Silvia Ayllón \\ Gabriel Escanés
}

La revista está indexada en los directorios:

LATINDEX, REDALYC, IRESIE, CLASE, DIALNET, DOAJ, E-REVIST@S,

La revista está incluida en los sitios:

REDIE, RINACE, OEI, MAESTROTECA, PREAL, HUASCARAN, CLASCO 


\title{
VARIABLES QUE INFLUYEN EN LA DESERCIÓN DE ESTUDIANTES UNIVERSITARIOS DE PRIMER AÑO. CONSTRUCCIÓN DE ÍNDICES DE RIESGO DE ABANDONO \\ VARIABLES THAT INFLUENCE FIRST YEAR UNIVERSITY STUDENTS' DROPOUT RATES.CONSTRUCTION OF DROPOUT RISK INDEXES
}

\author{
Aldo Merlino ${ }^{1}$ \\ Silvia Ayllón ${ }^{2}$ \\ Gabriel Escanés ${ }^{3}$
}

\begin{abstract}
Resumen: El presente artículo se enfoca en analizar la relación que existe entre la deserción de los estudios universitarios (durante el primer año cursado) y el comportamiento de ciertas variables específicas, medidas en los ingresantes a la Universidad Siglo 21 en Córdoba, Argentina. La investigación permitió desarrollar dos modelos de predicción del riesgo de abandono entre los estudiantes que ingresan a la universidad. Dichos modelos se denominan IRAP (Indice de Riesgo de Abandono Provisorio) e IRAD (Índice de Riesgo de Abandono Definitivo). Ambos se expresan en escalas de 5 categorías (desde riesgo muy bajo a muy alto). Los dos índices de riesgo se obtienen a partir de la aplicación de una serie de cuestionarios que miden diferentes variables con las cuales se construyen el IRAP y el IRAD. Ambos índices han sido desarrollados con el propósito de contar con herramientas de predicción de la deserción, lo cual permite trabajar sobre la prevención de la misma de modo anticipado. Entre las principales conclusiones que se obtuvieron en la investigación pueden señalarse la relación del rendimiento académico con la deserción y la capacidad predictiva del índice IRAD, dado que se observa que el $44 \%$ de los alumnos con riesgo alto y muy alto, no se reinscribieron en el segundo semestre de cursado.
\end{abstract}

Palabras clave: ABANDONO DE LOS ESTUDIOS UNIVERSITARIOS, RENDIMIENTO ACADÉMICO, MEDICIÓN DE RIESGO DE ABANDONO, ARGENTINA.

Abstract: The current article focuses on analyzing the existing relationship between the dropout in university studies (during the first year) and the behavior of certain specific variables measured in the freshmen at Universidad Siglo 21 (Córdoba, Argentina). The research findings presented in this article allowed the development of two models of predictability on the dropout risk among the students who start their university studies. Such models are called TDRI (temporary dropout risk index) and PDRI (permanent dropout risk index). Both are expressed in scales of 5 categories (from the lowest to the highest risk). Both risk indexes are obtained from the application of a series of questionnaires that measure different variables used in the TDRI and PDRI analysis. Both indexes have been developed with the purpose of having tools for dropout prediction, which allows to work on its prevention in an anticipated manner. Among the main conclusions obtained in the research, we can point out the relation between academic success, dropout and the PDRI, considering that the $44 \%$ of high-risk and very high risk students did not enroll in the second semester.

Key words: UNIVERSITY DROPOUT, ACADEMIC ACHIEVEMENT, DROPOUT RISK MEASUREMENT, ARGENTINA.

\footnotetext{
1 Psicólogo, Master en Sociosemiótica, Doctor en Semiótica, Decano del Departamento de Investigación, de la Universidad Siglo 21, Argentina, Profesor de la cátedra Seminario Final (investigación aplicada). Dirección electrónica: amerlino@uesiglo21.edu.ar

2 Psicóloga, Master en Metodología de la Investigación Científica, Especialista en Psicología clínica y educacional. Investigadora adjunta de la Universidad Siglo 21, Argentina. Dirección electrónica: sayllon@uesiglo21.edu.ar

${ }^{3}$ Licenciado en Comercialización, Maestrando y Doctorando en Demografía. Profesor adjunto de la cátedra de Investigación de Mercados, Investigador de la Universidad Siglo 21, Argentina. Dirección electrónica: gescanes@uesiglo21.edu.ar
}

Artículo recibido: 13 de marzo, 2011

Aprobado: 28 de julio, 2011 


\section{Introducción}

En general, el fenómeno de la deserción universitaria ha sido comprendido como un complejo proceso que obedece a múltiples causas, que conducen a que una importante cantidad de estudiantes inicien pero no completen sus estudios superiores. Esto produce una diferencia significativa entre el porcentaje de alumnos que ingresa y el porcentaje que egresa, lo cual constituye un problema para el individuo, para su grupo familiar, para las instituciones educativas y para los gobiernos, con sus correspondientes consecuencias psicológicas, sociales y económicas.

Los determinantes de dicha deserción pueden analizarse desde varias dimensiones, debido a la complejidad de los factores involucrados en el fenómeno. Algunos investigadores centran su atención en las características intrínsecas del individuo, mientras que otros se enfocan en factores externos al mismo, relacionados con la integración social a las instituciones y a la relación con sus miembros (Tinto, 1975, 1982, 1992, 2008; Bean, 1986; Bean y Eaton, 2003; Edel Navarro, 2003).

Por lo anterior, el presente artículo se concentra en analizar la relación que existe entre la deserción de los estudios universitarios (durante el primer año cursado) y el comportamiento de ciertas variables específicas medidas en los ingresantes a la Universidad siglo 21 (Córdoba, Argentina).

La investigación que se presenta en este artículo apuntó a desarrollar dos modelos de predicción del riesgo de abandono entre los estudiantes que ingresan a la universidad. Dichos modelos se denominarán IRAP (Índice de Riesgo de Abandono Provisorio) e IRAD (Índice de Riesgo de Abandono Definitivo). Ambos índices han sido desarrollados con el propósito de contar con herramientas de predicción de la deserción, lo cual permite trabajar sobre la prevención de la misma de modo anticipado.

Entonces, a partir de la medición del comportamiento de las variables comprendidas en los índices IRAP e IRAD, se intentará dar respuesta a la siguiente pregunta que constituye el problema central del trabajo de investigación: ¿Qué relación existe entre el comportamiento de las variables que componen el IRAP e IRAD y el comportamiento de la variable deserción? 


\subsection{Supuesto (hipótesis inicial) de trabajo}

A los fines de generar respuestas provisorias al establecimiento de la relación entre las variables mencionadas en la pregunta de investigación, se formuló el siguiente supuesto central, que funciona a modo de hipótesis general de trabajo:

Los alumnos ingresantes que presentan alguna de las siguientes características o una combinación de las mismas, a saber:

1.1.1 Niveles de rendimiento verbal calificados como regular, bajo o marginal.

1.1.2 Niveles (puntajes) bajos en responsabilidad.

1.1.3 Rendimiento académico bajo y muy bajo.

1.1.4 Bajo nivel de satisfacción con la Universidad Siglo 21.

1.1.5 Autopercepción como alumnos poco o muy poco motivados.

1.1.6 Autopercepción como alumnos que se encuentran retrasados en su estudio.

1.1.7 Calificación baja de los docentes.

presentarán mayores índices de deserción, que los alumnos que no presentan dichas características.

\subsection{Objetivos de la investigación}

Los objetivos generales del trabajo de investigación, cuyos resultados se presentan en este artículo, fueron:

1.2.1 Medir el comportamiento de las variables que componen el IRAP y el IRAD, en los alumnos ingresantes a primer año de las carreras universitarias que ofrece la Universidad Siglo 21.

1.2.2 Establecer correlaciones entre dichas variables y la variable deserción (abandono) de los estudios universitarios en el primer año de cursado.

\section{Metodología}

Para el desarrollo de la investigación, se implementó el siguiente diseño, expresado en la ficha técnica que sigue:

- Tipo de investigación: descriptiva, correlacional.

- Método: cuantitativo.

- Técnica de recolección de información: encuesta autoadministrada vía web. 
- Instrumento de recolección de datos: cuestionario estructurado, con respuestas de opción múltiple, aplicado en dos momentos del tiempo desde la inscripción del estudiante.

- Se realizó seguimiento del grupo en las siguientes instancias: ingreso (encuesta inicial), rendimiento (notas en los parciales); satisfacción durante el cursado del cuatrimestre (encuesta institucional) y listado de inscritos al iniciar el segundo cuatrimestre (registrando su re-inscripción o la falta de ésta).

- Población de estudio: estudiantes que ingresaron en el año 2010 a primer año de todas las carreras de grado ofrecidas por la Universidad Siglo 21, en la modalidad presencial (cursado en el campus).

- Criterio muestral: censo. Número de alumnos censados: 793.

\section{Breves antecedentes sobre el concepto de deserción}

En este apartado, y por una cuestión de extensión, contextualizaremos brevemente la noción de deserción y los factores que, en diferentes estudios, han sido asociadas a ella.

En la actualidad, no existe un consenso general sobre la definición de deserción en lo relativo a los estudios universitarios. El fenómeno de la deserción estudiantil universitaria ha sido un tema de investigación desde hace varias décadas (Ajzen y Fishbein, 1975; Spady, 1970; Hyun-Joo, 2003; Ishiyama, 2000; Rienks y Taylor, 2009) y es abordable desde diversos puntos de vista, que podrían organizarse en cinco grandes modelos: a) psicológicos, b) sociológicos, c) económicos, d) organizacionales y e) de interacción (Himmel, 2002).

En ese sentido, los modelos psicológicos señalan que existen diferencias entre los alumnos que terminan sus estudios universitarios respecto de aquellos que no logran finalizarlos. La diferencia se halla en los rasgos de la personalidad. La teoría de la Acción Razonada, desarrollada por Ajzen y Fishbein (1975), explica que el debilitamiento de las intenciones iniciales de los estudiantes en pos de concluir sus estudios es -en rasgos generales- lo que provoca la deserción. Esta teoría de la Acción Razonada es considerada un predictor (Breva Asensio y Carpi Ballester, 2002), un juicio probabilístico en el que puede estimarse la intención de la conducta directamente mediante una escala de probabilidad (Reyes Rodríguez, 2007). 
Por su parte, los modelos sociológicos consideran la influencia de factores externos al individuo, además de los rasgos de la personalidad (Díaz Peralta, 2008; Morris, 2002). Uno de sus pioneros, Spady (1970), se apoyó en el enfoque de Durkheim considerando a la deserción como semejante al suicidio en una sociedad y a las universidades como un sistema con determinados valores y estructura social. En este ámbito, bajos niveles de integración social aumentan la probabilidad de desertar. Entre los predictores de la deserción, el autor señala la integración social, la integración académica, el género y el promedio de notas en las materias, entre otros.

Los modelos económicos plantean que si la población estudiantil universitaria percibe mayores beneficios sociales y económicos asociados a los estudios universitarios que los derivados de otras actividades, como el trabajo, entonces, la población decide permanecer en la universidad, en vez de abandonar sus estudios. Este enfoque hace referencia al costo/beneficio que evalúa el estudiante.

Los modelos organizacionales explican que la deserción se encuentra en función de las cualidades de las universidades en cuanto a la integración social. Además, ponen el acento en la integración del estudiante a la institución, sentirse parte de la misma, y las experiencias vividas en relación con la calidad docente y al proceso de aprendizaje en el aula, en relación con el contacto con los docentes durante el cursado de toda su carrera.

Por último, los modelos de interacción explican la permanencia en los estudios en función del grado de ajuste entre el estudiante y la institución. Como explica Tinto $(1975,1997,2008)$, las experiencias académicas previas al acceso universitario, las vivencias durante su permanencia en sus estudios de grado y las experiencias de integración social (donde influyen las características personales de los estudiantes), explican la deserción o permanencia de un estudiante. Mientras tanto, Bean (1980) agrega al modelo de Tinto el concepto de satisfacción de los estudiantes con 4 factores vinculados a la vida académica: a) académicos, b) psicosociales, c) ambientales y d) de socialización.

Si avanzamos hacia algunas definiciones específicas del concepto de deserción, encontramos la que proponen Teresa Chalabe, Lilia Pérez y Elizabeth Truninger, a saber: "el término deserción es utilizado para describir el comportamiento de los alumnos que abandonaron los estudios, sin reparar en las razones o circunstancias que determinaron ese abandono" (2004, s.p.). 
Por su parte, Erika Himmel explica que: "la deserción se refiere al abandono prematuro de un programa de estudios antes de alcanzar el título o grado, y comprende un tiempo suficientemente largo como para descartar la posibilidad de que el estudiante se reincorpore" (2002, p. 94). La autora agrega que la deserción puede clasificarse en voluntaria e involuntaria. La primera se refiere a la renuncia a la carrera por parte del estudiante o del abandono no informado a la institución de educación superior, mientras que la segunda es consecuencia de una decisión institucional, basada en sus reglamentos vigentes, que impone al estudiante a retirarse de los estudios.

En nuestro trabajo de investigación utilizamos el término deserción para referirnos, específicamente, a la situación en la que un alumno de la Universidad Siglo 21 -matriculado en su primer semestre de cursado- opta por interrumpir, de manera permanente o transitoria, sus estudios superiores, antes de que comience el semestre siguiente. Los datos de la interrupción se obtienen de la comparación entre listados de alumnos matriculados en los dos períodos consecutivos (semestre 1 y semestre 2).

\section{Análisis y resultados de la investigación}

A través de la investigación desarrollada se intenta identificar qué factores inciden en la deserción y estimar la importancia o peso relativo de cada factor en la predicción de la deserción estudiantil de los alumnos ingresantes del primer semestre de 2010, en la Universidad Siglo 21.

En ese sentido, se considera alumno ingresante al estudiante que ingresó a primer año de alguna de las carreras en la modalidad presencial. A tales fines, y a partir de un estudio exploratorio desarrollado entre los años 2008 y 2009 en la Universidad Siglo 21, se identificaron las siguientes variables que potencialmente pueden influir en la deserción de los estudios universitarios:

- Habilidades verbales (razonamiento verbal): se entiende por habilidades verbales la capacidad del estudiante para entender conceptos formulados en palabras. Se evalúa la capacidad para abstraer o generalizar y pensar constructivamente, más que la simple fluidez verbal o el reconocimiento de un vocabulario. Se analiza -a partir de la administración de un test específico- el razonamiento por analogía, uno 
de los tipos de razonamiento más complejos. El test aplicado es el DAT (Test de Aptitudes Diferenciales) de Bennett, Seashore y Wesman (1997), en su versión T.

- Grado de responsabilidad: esta variable corresponde al conjunto de rasgos de personalidad (comportamientos estables y duraderos en el individuo a lo largo del tiempo), que implican coherencia, organización, perseverancia y posibilidades de sostener el esfuerzo en la actividad académica,

- Rendimiento académico: se refiere al nivel de conocimientos demostrado con respecto a los contenidos en una materia en relación a normas de evaluación preestablecidas.

- Autopercepción como alumno: consiste en el autorregistro que tiene el alumno de su ritmo de estudio y de su motivación.

- Satisfacción con la universidad: se refiere al grado de conformidad y de satisfacción general de sus necesidades que experimenta el alumno en relación a la Universidad Siglo 21. Las dos dimensiones para la construcción de esta variable son satisfacción e intención de re-elección de la misma universidad.

- Evaluación de los docentes: esta variable se construye con tres dimensiones, que el alumno evalúa en sus docentes: comprensión de contenidos, clima de trabajo y respeto del docente por horarios de clase.

Todas las variables mencionadas se agrupan en dos índices, cada uno de los cuales es obtenido en función de la recolección de datos de los alumnos ingresantes en dos momentos de su vida estudiantil, estos son:

A. Índice de Riesgo de Abandono Provisorio (IRAP): valor que conjuga los datos obtenidos en las variables:

- DAT (test de rendimiento en habilidades verbales).

- Responsabilidad.

Este índice es obtenido antes de que el alumno comience con el ciclo lectivo, mediante la aplicación de las pruebas (tests) vía web. Por ello, el IRAP se constituye en un índice que predice el abandono de manera temprana, pero con menos precisión y de modo provisorio. 
B. Índice de Riesgo de Abandono Definitivo (IRAD): valor numérico que conjuga los datos obtenidos en las variables:

- Rendimiento académico.

- Autopercepción como alumno.

- Satisfacción con la Universidad Siglo 21.

- Evaluación de los docentes.

- IRAP.

Para obtener este índice, es necesario que el alumno termine su ciclo lectivo (semestral), porque es a partir de la regularización (aprobación) de las materias que cursa, que se obtiene el dato de la variable rendimiento académico, que resulta central en el índice IRAD.

\subsection{Desarrollo de la variable IRAP (índice de riesgo de abandono provisorio)}

A continuación, se describirá el proceso de desarrollo y las mediciones llevadas a cabo para obtener el índice IRAP, que se propone como un índice de medición del riesgo que un alumno presenta respecto del abandono prematuro de sus estudios universitarios.

La primera variable que se incorpora en el modelo de predicción que presentamos es la responsabilidad. A fin de medir esta variable se desarrolló una serie de nueve proposiciones a las que el estudiante ingresante debe responder indicando cuánto se identifica con dichas proposiciones en una escala numérica del 1 al 10. El 1 significa que la frase no describe en absoluto cómo el estudiante se ve a sí mismo, mientras que 10 significa que la frase lo describe justo cómo se percibe. El promedio obtenido entre todas las respuestas representa el grado de responsabilidad (auto percibido) del estudiante.

La elección de los reactivos utilizados se basó en el test conocido como Inventario de Personalidad NEO PI-R, creado por Paul Costa y Robert McCrae (1999). Las siglas NEO hacen referencia a los tres primeros factores de personalidad que se tomaron en cuenta (Neuroticismo, Extraversión y Apertura, por sus siglas en inglés) a los que luego se añadieron $A$ (Amabilidad) y C (Responsabilidad). Sobre este último factor se enfocaron las mediciones en la presente investigación.

Cabe aclarar que, a partir de una prueba piloto desarrollada por los propios investigadores y a fin de evitar caer en la redundancia, se decidió eliminar algunos reactivos que carecían de 
relevancia dadas las respuestas básicamente idénticas a las obtenidas en los ítems conservados.

Para Costa y McCrae (1999), la responsabilidad refiere al grado de organización del individuo, su perseverancia y la motivación dirigida a un objetivo. En este punto se consideran 6 componentes: competencia (sentimiento de capacidad), orden (mantenimiento de cierto ordenamiento), sentido del deber (concientización de las acciones), necesidades de logro (aspiraciones y objetivos), autodisciplina (realización completa de actividades) y, por último, deliberación (pensamiento previo a la actuación).

El criterio considerado para la elección de las frases fue definido por la variabilidad que muestran las mismas en relación con la media. Es decir, los 9 ítems fueron incorporados al modelo por la potencialidad de diferenciar a los alumnos en su grado de responsabilidad. Las proposiciones utilizadas en la medición, junto con sus correspondientes coeficientes de variación, se mencionan a continuación:

- En mi vida, siempre me fijo metas para cumplir. (CV: 0.312).

- Si se me interponen muchos obstáculos en algo que quiero hacer, tiendo a dejarlo. (CV: 0.35).

- Tengo muy claro lo que quiero lograr en cada etapa de mi vida. (CV: 0.311).

- Me gusta ponerme desafíos a mí mismo, para tratar de lograrlos. (CV: 0.296).

- Soy una persona que planifica mucho para el futuro. (CV: 0.322).

- Muchas veces, dejo las cosas que debiera hacer hoy, para mañana. (CV: 0.509).

- Antes de hacer algo, siempre pienso en las consecuencias. (CV: 0.419).

- Suele sucederme que, cuando tengo que hacer algo, necesito que alguien me lo recuerde, porque suelo olvidarme. (CV: 0.452).

- Suelo atrasarme en la entrega de mis trabajos. (CV: 0.296).

Los resultados que se obtuvieron en la medición de la responsabilidad arrojan los siguientes datos: 
Tabla No. 1

Responsabilidad.

\begin{tabular}{|c|c|c|}
\hline & Frecuencia & Porcentaje \\
\hline Puntaje Alto & 297 & $37,5 \%$ \\
\hline Puntaje Medio & 469 & $59,1 \%$ \\
\hline Puntaje Bajo & 27 & $3,4 \%$ \\
\hline Total & 793 & $100,0 \%$ \\
\hline \multicolumn{2}{|r|}{ Fuente: Elaboración propia } \\
\end{tabular}

A partir de los resultados obtenidos se comparó el comportamiento de la variable responsabilidad con el de la variable abandono (no rematriculación al siguiente semestre), obteniéndose los siguientes resultados:

Tabla No. 2

Comparación de los niveles de Responsabilidad según condición de reinscripción.

\begin{tabular}{|c|c|c|}
\hline & Con Reinscripción & Sin Reinscripción \\
\hline Puntaje Alto & $37,8 \%$ & $35,5 \%$ \\
\hline Puntaje Medio & $59,1 \%$ & $59,5 \%$ \\
\hline Puntaje Bajo & $3,1 \%$ & $5,0 \%$ \\
\hline Total & $100,0 \%$ & $100,0 \%$ \\
\hline
\end{tabular}

Fuente: Elaboración propia

Como se observa en la tabla 2, no aparecen diferencias sustanciales en la conducta de abandono dado que tanto los alumnos que se reinscribieron como los que no lo hicieron presentan similares distribuciones de frecuencia en la variable responsabilidad.

Como segunda variable constitutiva del índice de riesgo de abandono provisorio (IRAP) se incorporó una variable vinculada al Test de Aptitudes Diferenciales DAT (Forma T). EI DAT es una batería de ocho sub-tests que evalúan distintas aptitudes y que presenta la ventaja de poder ser administrado en forma total o parcial, dependiendo de los objetivos de la evaluación.

Por lo tanto, el análisis de las puntuaciones puede realizarse para cada test por separado o integrando la información que resulta de la evaluación de los puntajes de dos o más tests. El 
sub-test seleccionado fue el de Razonamiento Verbal dada la elevada correlación con el rendimiento académico según estudios previos realizados en la Universidad Siglo 21.

La variable del test DAT incorporada al IRAP es la denominada razonamiento verbal. Los resultados del test DAT en la variable mencionada fueron los siguientes:

Tabla No. 3

Segmentos D.A.T.

\begin{tabular}{|c|c|c|}
\hline & Frecuencia & Porcentaje \\
\hline Puntaje Muy Bueno & 78 & $9,8 \%$ \\
\hline Puntaje Bueno & 129 & $16,3 \%$ \\
\hline Puntaje Regular & 251 & $31,7 \%$ \\
\hline Puntaje Bajo & 188 & $23,7 \%$ \\
\hline Puntaje Marginal & 147 & $18,5 \%$ \\
\hline Total & 793 & $100,0 \%$ \\
\hline
\end{tabular}

Fuente: Elaboración propia

Como se observa en la tabla 3, el puntaje obtenido por los alumnos se divide en cinco categorías, que reflejan la cantidad de respuestas correctas obtenidas en el test. Tal como puede observarse, la mayor cantidad de alumnos se concentra en el puntaje regular.

Si avanzamos hacia la puesta en relación de la variable puntaje en el test DAT con la variable abandono o deserción (representada por la reinscripción o no), encontramos los siguientes resultados:

Tabla No. 4

Comparación de los Segmentos D.A.T. según condición de reinscripción.

\begin{tabular}{|c|c|c|}
\hline & Con Reinscripción & Sin Reinscripción \\
\hline Puntaje Muy Bueno & $10,6 \%$ & $5,8 \%$ \\
\hline Puntaje Bueno & $15,8 \%$ & $19,0 \%$ \\
\hline Puntaje Regular & $33,6 \%$ & $20,7 \%$ \\
\hline Puntaje Bajo & $22,5 \%$ & $30,6 \%$ \\
\hline Puntaje Marginal & $17,6 \%$ & $24,0 \%$ \\
\hline Total & $100,0 \%$ & $100,0 \%$ \\
\hline
\end{tabular}

Fuente: Elaboración propia 
Como puede observarse en la tabla precedente, entre los alumnos que no se reinscribieron, encontramos mayor porcentaje de resultados calificados como bajo o marginal (54,6\% combinando ambos puntajes), mientras que entre los alumnos que sí se reinscribieron, la combinación de ambos puntajes alcanza algo más del 43\%, lo que representa 10 puntos porcentuales menos. Estos resultados muestran una relación -por lo menos incipiente- entre la variable puntaje DAT y la variable abandono.

Finalmente, se combinan ambas variables medidas (puntaje DAT y Responsabilidad) para obtener el IRAP. La ecuación a partir de la cual se obtiene el Índice de Riesgo de Abandono Provisorio (IRAP) se muestra a continuación:

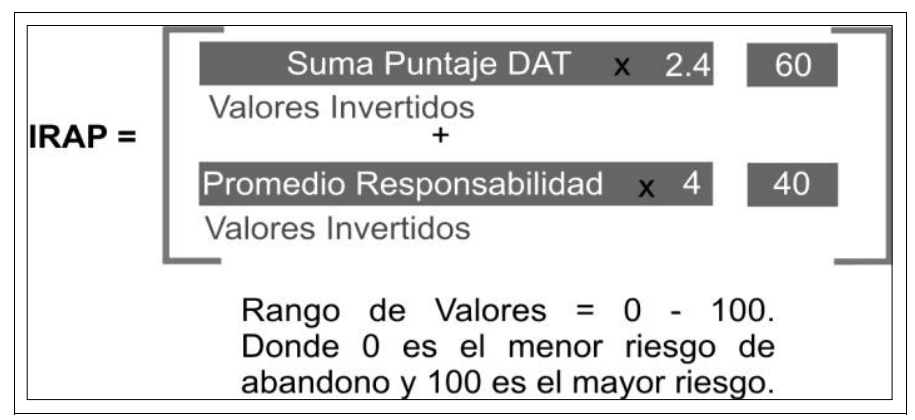

Figura No. 1. Índice de Riesgo de Abandono Provisorio

Los puntajes IRAP obtenidos por los alumnos ingresantes, se reflejan en la siguiente tabla:

Tabla No. 5

Índice de Riesgo de Abandono Provisorio

(categorías equidistantes).

\begin{tabular}{|c|c|c|}
\hline & Frecuencia & Porcentaje \\
\hline Riesgo Muy Alto & 2 & $0,3 \%$ \\
\hline Riesgo Alto & 98 & $12,4 \%$ \\
\hline Riesgo Medio & 401 & $50,6 \%$ \\
\hline Riesgo Bajo & 251 & $31,7 \%$ \\
\hline Riesgo Muy Bajo & 41 & $5,2 \%$ \\
\hline Total & 793 & $100,0 \%$ \\
\hline
\end{tabular}

Fuente: Elaboración propia 
Como se observa en la tabla, la mayoría de los alumnos se ubica en un riesgo medio. Si se relacionan los puntajes IRAP obtenidos con la variable abandono, observaremos los siguientes resultados:

Tabla No. 6

Comparación de los segmentos de riesgos de abandono según condición de reinscripción.

\begin{tabular}{|c|c|c|}
\hline & Con Reinscripción & Sin Reinscripción \\
\hline Riesgo Muy Alto & $0,3 \%$ & $0,0 \%$ \\
\hline Riesgo Alto & $10,6 \%$ & $22,3 \%$ \\
\hline Riesgo Medio & $50,3 \%$ & $52,1 \%$ \\
\hline Riesgo Bajo & $33,2 \%$ & $23,1 \%$ \\
\hline Riesgo Muy Bajo & $5,7 \%$ & $2,5 \%$ \\
\hline Total & $100,0 \%$ & $100,0 \%$ \\
\hline
\end{tabular}

Fuente: Elaboración propia

Tal como se observa en la tabla 6 , el $22,3 \%$ de los alumnos que no se reinscribieron presentaba un riesgo catalogado como alto, mientras que entre los alumnos reinscriptos el riesgo alto baja doce puntos porcentuales. Esto refleja que los estudiantes desertores presentan mayor índice de Riesgo de Abandono Provisorio (IRAP). En otros términos, el conocimiento de la variable IRAP permite predecir, con cierto grado de acierto, el comportamiento de la reinscripción.

Como se podrá observar más delante en la lectura del artículo, el IRAP tiene menor precisión en cuanto a sus predicciones en relación al IRAD, debido al momento tan preliminar en el cual se realiza la cuantificación. Sin embargo, se observan más los alumnos que no se reinscriben y que han sido señalados como de riesgo alto (22.3\% vs. $10.6 \%$ ) y más los que se reinscriben que han sido señalados como de riesgo bajo o muy bajo (33.2\% vs $23.1 \%$; y $5.7 \%$ vs $2.5 \%$, respectivamente).

\subsection{Desarrollo del índice de riesgo de abandono definitivo (IRAD)}

Como siguiente etapa del trabajo de investigación, se desarrolló un segundo Índice de Riesgo de Abandono (IRAD), formado a partir de variables que implican -necesariamente- el 
paso del alumno por el semestre, algo que no sucedía con el IRAP, que es considerado un índice de riesgo temprano, y por ello menos preciso.

La primera variable que se incluye en el IRAD es el rendimiento académico. Esta variable está construida sobre una base bidimensional, a saber:

A. Cantidad de materias regularizadas (aprobadas) por los alumnos.

B. Notas (promedio) en las evaluaciones institucionales de las materias regularizadas en el semestre. Incluye la nota de las evaluaciones recuperatorias.

Véase la siguiente figura que muestra ambas dimensiones del Rendimiento Académico.

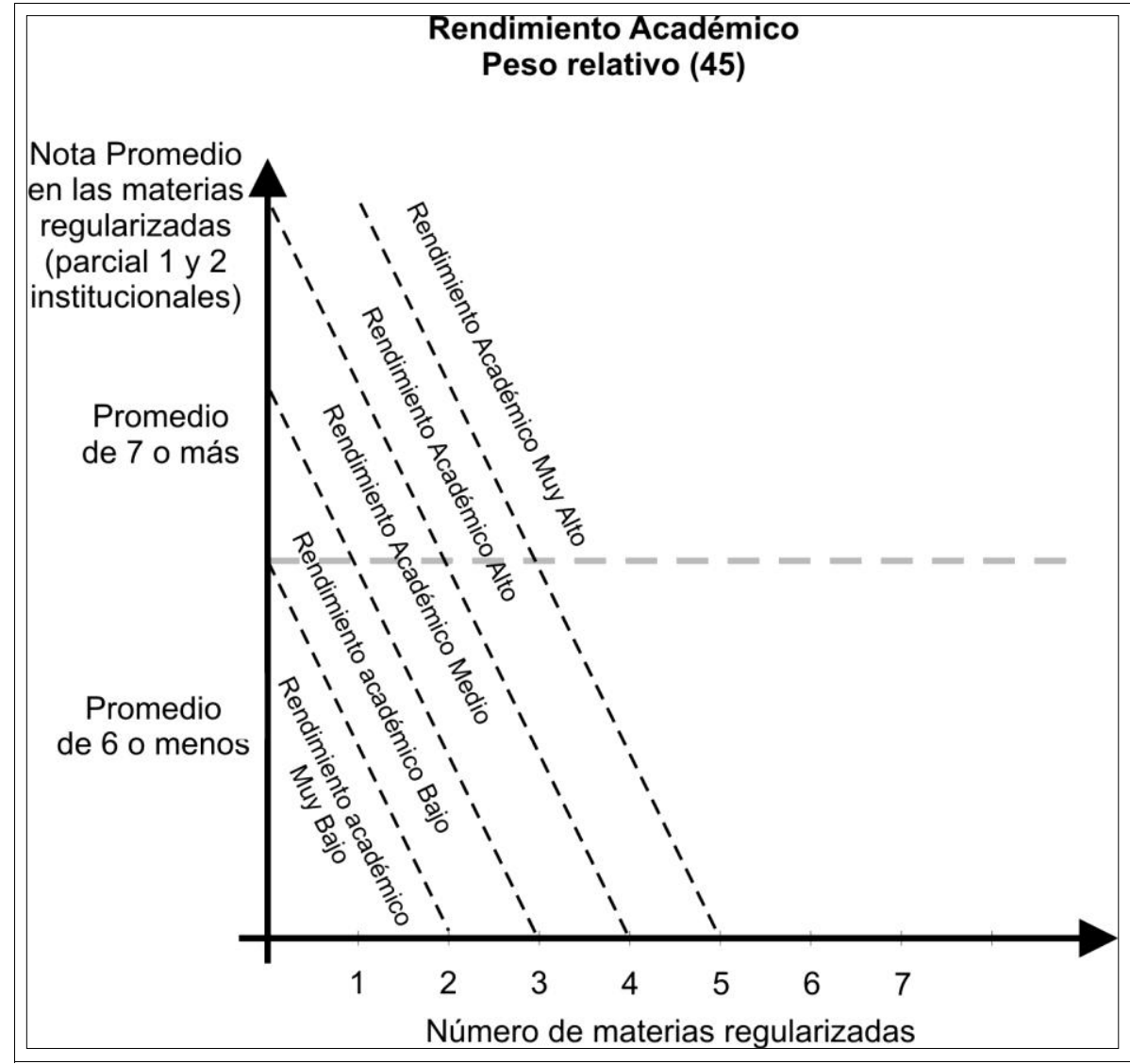

Figura No. 2. Rendimiento académico

Entre ambas dimensiones surgen cinco categorías de la variable: 
- Rendimiento muy alto: cinco o más materias regularizadas (con nota promedio 7 o más).

- Rendimiento alto: cuatro materias regularizadas (con nota promedio 7 o más) 0 cinco más materias regularizadas (con promedio de notas menor o igual a 6).

- Rendimiento medio: tres materias regularizadas (con nota promedio 70 más) 0 cuatro materias regularizadas (con promedio de notas menor o igual a 6).

- Rendimiento bajo: dos materias regularizadas (con nota promedio 7 o más) o tres materias regularizadas (con promedio de notas menor o igual a 6).

- Rendimiento muy bajo: hasta una materia regularizada (sin importar la nota promedio) o dos materias regularizadas (con promedio de notas menor o igual a 6).

A continuación se presentan los resultados obtenidos en la variable rendimiento académico, en los alumnos ingresantes:

Tabla No. 7.

Rendimiento Académico.

\begin{tabular}{|c|c|c|}
\hline & Frecuencia & Porcentaje \\
\hline Rendimiento Académico Muy Alto & 149 & $18,8 \%$ \\
\hline Rendimiento Académico Alto & 168 & $21,2 \%$ \\
\hline Rendimiento Académico Medio & 152 & $19,2 \%$ \\
\hline Rendimiento Académico Bajo & 149 & $18,8 \%$ \\
\hline Rendimiento Académico Muy Bajo & 175 & $22,1 \%$ \\
\hline Total & 793 & $100,0 \%$ \\
\hline
\end{tabular}

Fuente: Elaboración propia

Como se observa en la tabla anterior, la distribución de frecuencias en las cinco categorías de rendimiento académico es pareja.

Ahora bien, si cruzamos la variable rendimiento académico con la variable abandono, observaremos que comienza a vislumbrarse una de las relaciones más sólidas que arroja el estudio. 
Tabla No. 8

Comparación de los segmentos de rendimiento académico según condición de reinscripción.

\begin{tabular}{|c|c|c|}
\hline & Con Reinscripción & Sin Reinscripción \\
\hline Rendimiento Académico Muy Alto & $21,6 \%$ & $3,3 \%$ \\
\hline Rendimiento Académico Alto & $23,1 \%$ & $10,7 \%$ \\
\hline Rendimiento Académico Medio & $20,5 \%$ & $11,6 \%$ \\
\hline Rendimiento Académico Bajo & $17,9 \%$ & $24,0 \%$ \\
\hline Rendimiento Académico Muy Bajo & $17,0 \%$ & $50,4 \%$ \\
\hline Total & $100,0 \%$ & $100,0 \%$ \\
\hline
\end{tabular}

Fuente: Elaboración propia

Como puede observarse, el $74 \%$ de los que no se reinscribieron al semestre posterior, presentaban un rendimiento académico bajo y muy bajo (combinado), mientras que entre quienes sí se reinscribieron, dicho porcentaje combinado llega al 34,9\%, lo que significa casi 40 puntos menos que en el caso de los que abandonaron sus estudios. Encontramos así que la variable rendimiento académico se presentará como la más fuertemente asociada al abandono de los estudios universitarios en el primer año de cursado

La segunda variable que se incluye en el modelo IRAD se refiere a la autopercepción como alumno. Este indicador se construye sobre otra base bidimensional:

A. Estado del cursado (cuán al día percibe el alumno que se encuentra en relación al cursado de las materias).

B. Motivación para el estudio (cuán fuerte es la fuerza interna que lo impulsa hacia el estudio).

Véase la siguiente figura que muestra ambas dimensiones de la variable. 


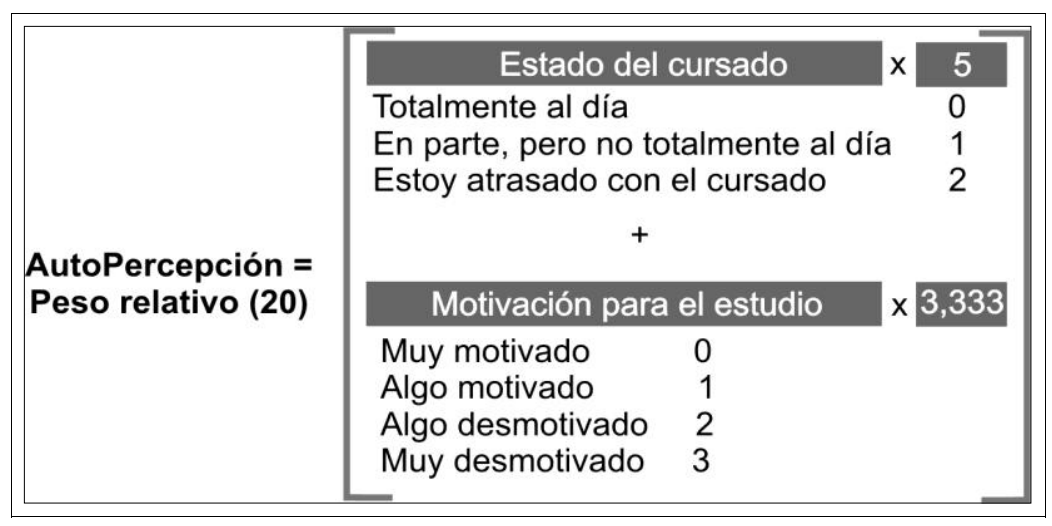

Figura No. 3. Autopercepción como alumno

Los resultados en el puntaje de autopercepción se expresan como sigue:

Tabla No. 9

Cuán motivado está usted para estudiar.

\begin{tabular}{|c|c|c|}
\hline & Frecuencia & Porcentaje \\
\hline Muy motivado & 343 & $43,3 \%$ \\
\hline Algo motivado & 371 & $46,8 \%$ \\
\hline Algo desmotivado & 59 & $7,4 \%$ \\
\hline Muy desmotivado & 20 & $2,5 \%$ \\
\hline Total & 793 & $100,0 \%$ \\
\hline
\end{tabular}

Fuente: Elaboración propia

Como puede observarse, la autopercepción de los alumnos se distribuye mayoritariamente entre las opciones muy motivado y algo motivado.

Cuando cruzamos la variable percepción de motivación con abandono, observamos lo siguiente: 
Tabla No. 10

Comparación de la motivación para estudiar según condición de reinscripción.

\begin{tabular}{|c|c|c|}
\hline & Con Reinscripción & Sin Reinscripción \\
\hline Muy motivado & $44,8 \%$ & $34,7 \%$ \\
\hline Algo motivado & $46,7 \%$ & $47,1 \%$ \\
\hline Algo desmotivado & $6,4 \%$ & $13,2 \%$ \\
\hline Muy desmotivado & $2,1 \%$ & $5,0 \%$ \\
\hline Total & $100,0 \%$ & $100,0 \%$ \\
\hline
\end{tabular}

Fuente: Elaboración propia

Si bien los porcentajes tienden a mantenerse, se nota una disminución de 10 puntos porcentuales en la alta motivación (alumnos muy motivados) entre quienes no se reinscribieron. También se observan puntos porcentuales más en la opción algo desmotivado.

Ahora, aun cuando los datos no son concluyentes, muestran cierta diferencia en lo relativo a la motivación, entre alumnos que se reinscribieron y aquellos que no lo hicieron, esto es: en el grupo de los que no se reinscribieron hay menos alumnos muy motivados $(10 \%$ menos que en el grupo de los reinscriptos) y más alumnos algo desmotivados y desmotivados (10\% más que en el grupo de los reinscritos). A nivel de hipótesis, puede pensarse que la motivación es un aspecto que puede coadyuvar a la deserción, en la medida en que se combine con otros que lo refuercen.

Dentro de la variable autopercepción como alumno también se tomó en cuenta cómo se percibe el estudiante en cuanto a los avances de sus estudios, es decir, cuan al día está con las materias que está cursando. Al respecto, observaremos, en las dos tablas que siguen, una tendencia similar a la que se notó en la percepción de motivación.

Tabla No. 11

Percepción de avance en sus estudios.

\begin{tabular}{|c|c|c|}
\hline & Frecuencia & Porcentaje \\
\hline Totalmente al día & 411 & $51,8 \%$ \\
\hline En parte, pero no totalmente al día & 343 & $43,3 \%$ \\
\hline Estoy atrasado con el cursado & 39 & $4,9 \%$ \\
\hline Total & 793 & $100,0 \%$ \\
\hline
\end{tabular}

Fuente: Elaboración propia 
Tabla No. 12

Comparación de la percepción de avance en sus estudios según condición de reinscripción.

\begin{tabular}{|c|c|c|}
\hline & Con Reinscripción & Sin Reinscripción \\
\hline Totalmente al día & $53,6 \%$ & $42,1 \%$ \\
\hline En parte, pero no totalmente al día & $42,7 \%$ & $46,3 \%$ \\
\hline Estoy atrasado con el cursado & $3,7 \%$ & $11,6 \%$ \\
\hline Total & $100,0 \%$ & $100,0 \%$ \\
\hline
\end{tabular}

Fuente: Elaboración propia

Se observa que hay menos alumnos totalmente al día dentro del grupo de los no reinscriptos (10\% menos). Este dato resulta pertinente si se aúna con el del rendimiento académico, que es la variable más fuertemente asociada con el abandono. Esto es, en la medida que el alumno no presenta un rendimiento adecuado, se atrasa en su cursado y, por lo tanto, se torna más propenso a desertar.

La tercera variable que se incluye en el modelo refiere a la satisfacción general con la Universidad Siglo 21. Este indicador se construye sobre otra base bidimensional:

A. Grado de satisfacción respecto de la Universidad Siglo 21.

B. Intención de re-elección de la Universidad Siglo 21.

Véase la siguiente figura que muestra ambas dimensiones de la satisfacción.

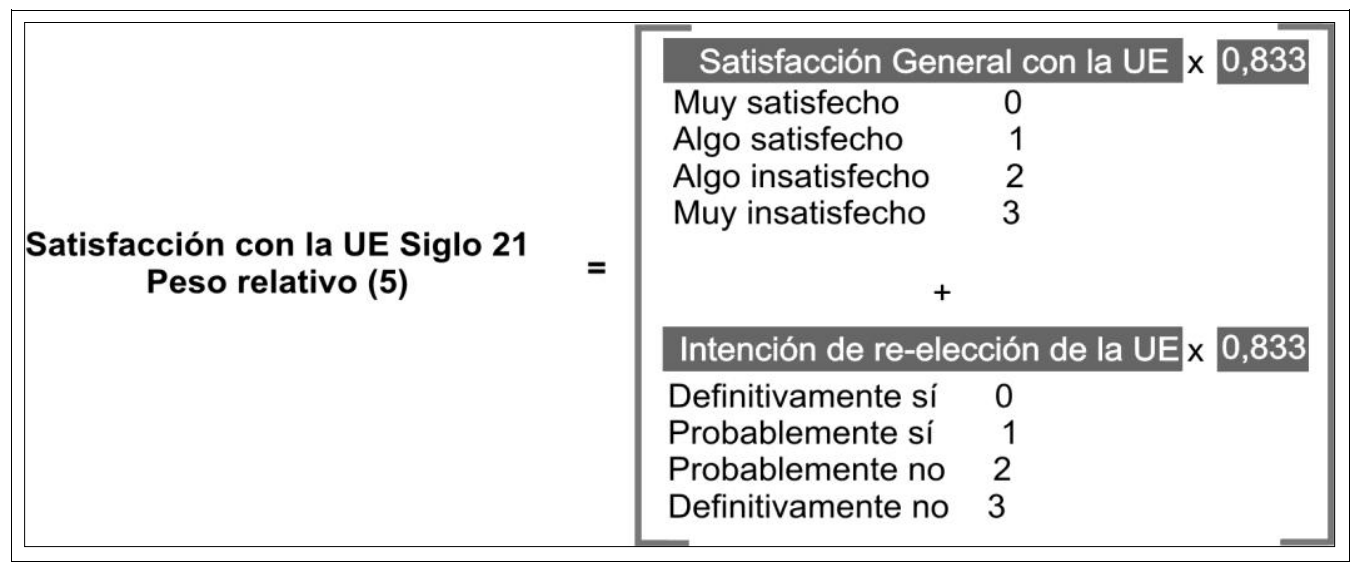

Figura No. 4. Satisfacción con la Universidad Siglo 21 
Se observa que la mayoría de los alumnos se encuentra en elevados niveles de satisfacción.

Tabla No. 13

Satisfacción con la Universidad Siglo 21.

\begin{tabular}{|c|c|c|}
\hline & Frecuencia & Porcentaje \\
\hline Muy satisfecho & 252 & $31,8 \%$ \\
\hline Satisfecho & 510 & $64,3 \%$ \\
\hline Insatisfecho & 23 & $2,9 \%$ \\
\hline Muy insatisfecho & 8 & $1,0 \%$ \\
\hline Total & 793 & $100,0 \%$ \\
\hline
\end{tabular}

Fuente: Elaboración propia

Y no se observa relación entre esta variable y el abandono, dado que la diferencia porcentual, entre los alumnos que se reinscribieron y los que no lo hicieron, muestra niveles de satisfacción similares para ambos grupos. En otros términos, la diferencia porcentual entre estos grupos no permite afirmar que los alumnos que se encuentran insatisfechos muestren comportamientos diferentes en su condición de reinscripción.

Tabla No. 14

Comparación de la satisfacción con la Universidad Siglo 21 según condición de reinscripción.

\begin{tabular}{|c|c|c|}
\hline & Con Reinscripción & Sin Reinscripción \\
\hline Muy satisfecho & $32,3 \%$ & $28,9 \%$ \\
\hline Satisfecho & $64,4 \%$ & $63,6 \%$ \\
\hline Insatisfecho & $2,5 \%$ & $5,0 \%$ \\
\hline Muy insatisfecho & $0,7 \%$ & $2,5 \%$ \\
\hline Total & $100 \%$ & $100 \%$ \\
\hline
\end{tabular}

Fuente: Elaboración propia

Lo mismo sucede con la otra dimensión de la variable, la intención de reinscribirse nuevamente si comenzara (hipotéticamente) de nuevo sus estudios 
Tabla No. 15

Intención de reinscripción.

\begin{tabular}{|c|c|c|}
\hline & Frecuencia & Porcentaje \\
\hline Definitivamente sí & 360 & $45,4 \%$ \\
\hline Probablemente sí & 388 & $48,9 \%$ \\
\hline Probablemente no & 37 & $4,7 \%$ \\
\hline Definitivamente no & 8 & $1,0 \%$ \\
\hline Total & 793 & $100,0 \%$ \\
\hline
\end{tabular}

Fuente: Elaboración propia

Tabla No.16

Comparación de la intención de reinscripción según condición de reinscripción.

\begin{tabular}{|c|c|c|}
\hline & Con Reinscripción & Sin Reinscripción \\
\hline Definitivamente sí & $45,5 \%$ & $44,6 \%$ \\
\hline Probablemente sí & $49,3 \%$ & $47,1 \%$ \\
\hline Probablemente no & $4,3 \%$ & $6,6 \%$ \\
\hline Definitivamente no & $0,9 \%$ & $1,7 \%$ \\
\hline Total & $100 \%$ & $100 \%$ \\
\hline
\end{tabular}

Fuente: Elaboración propia

La cuarta variable que se incluye en el modelo IRAD se refiere a la evaluación de los docentes. Este indicador se construye en base a tres dimensiones:

- Comprensión de contenidos explicados por el profesor en clases.

- Clima de trabajo en clases (posibilidad de opinar y participar en las clases).

- Respeto por los horarios de clases (puntualidad del docente).

Véase la siguiente figura que muestra las tres dimensiones de la variable. 


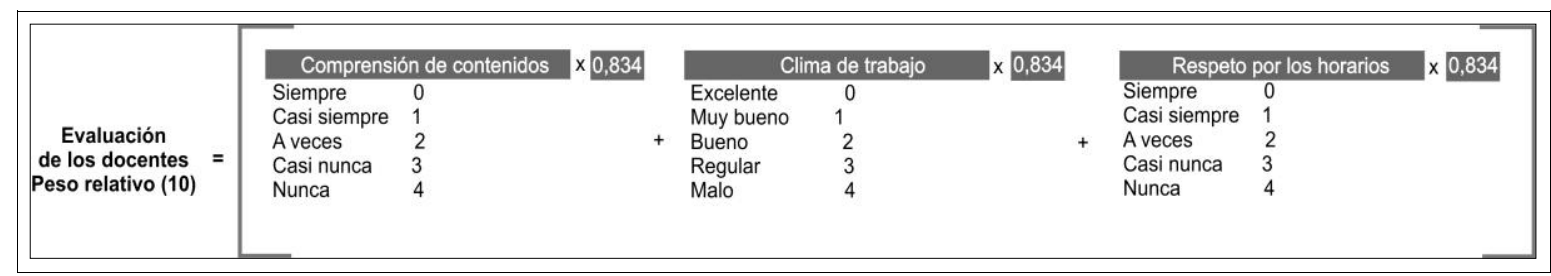

Figura No. 5. Evaluación de los docentes

Dado que las tres interrogantes son medidas para cada docente y materia, se tomará un promedio de los valores obtenidos de acuerdo con la cantidad de materias cursadas por el alumno.

Las tablas que siguen son frecuencias que muestran las preguntas de la evaluación docente. Debe tenerse en cuenta que la tablas no corresponden a una materia determinada, ni a un docente específico, sino a una combinación de materias y docentes. En cuanto a la primera dimensión se observa lo siguiente:

Tabla No.17

Comprendo lo que el docente explica en clase.

\begin{tabular}{|c|c|c|}
\hline & Frecuencia & Porcentaje \\
\hline Siempre & 454 & $57,3 \%$ \\
\hline Casi siempre & 238 & $30,0 \%$ \\
\hline A veces & 86 & $10,8 \%$ \\
\hline Casi nunca & 10 & $1,3 \%$ \\
\hline Nunca & 5 & $0,6 \%$ \\
\hline Total & 793 & $100,0 \%$ \\
\hline
\end{tabular}

Fuente: Elaboración propia 
Tabla No. 18

Comparación de la comprensión de lo que el docente explica en clases según condición de reinscripción.

\begin{tabular}{|c|c|c|}
\hline & Con Reinscripción & Sin Reinscripción \\
\hline Siempre & $48,4 \%$ & $49,5 \%$ \\
\hline Casi siempre & $31,0 \%$ & $31,2 \%$ \\
\hline A veces & $17,1 \%$ & $13,8 \%$ \\
\hline Casi nunca & $2,4 \%$ & $3,7 \%$ \\
\hline Nunca & $1 \%$ & $2 \%$ \\
\hline Total & $100 \%$ & $100 \%$ \\
\hline
\end{tabular}

Fuente: Elaboración propia

En relación con la segunda dimensión mencionada, es posible observar que:

Tabla No. 19

El clima de trabajo en el aula es...

\begin{tabular}{|c|c|c|}
\hline & Frecuencia & Porcentaje \\
\hline Excelente & 356 & $44,9 \%$ \\
\hline Muy Bueno & 305 & $38,5 \%$ \\
\hline Bueno & 99 & $12,5 \%$ \\
\hline Regular & 26 & $3,3 \%$ \\
\hline Malo & 7 & $0,9 \%$ \\
\hline Total & 793 & $100,0 \%$ \\
\hline \multicolumn{2}{|c}{} \\
\hline
\end{tabular}

Tabla No. 20

Comparación del clima de trabajo en el aula según condición de reinscripción.

\begin{tabular}{|c|c|c|}
\hline & Con Reinscripción & Sin Reinscripción \\
\hline Excelente & $46,1 \%$ & $42,3 \%$ \\
\hline Muy Bueno & $38,7 \%$ & $41,4 \%$ \\
\hline Bueno & $11,2 \%$ & $10,8 \%$ \\
\hline Regular & $3,1 \%$ & $4,5 \%$ \\
\hline Malo & $1 \%$ & $1 \%$ \\
\hline Total & $100 \%$ & $100 \%$ \\
\hline
\end{tabular}

Fuente: Elaboración propia 
Y en el último punto, vinculado con la puntualidad del docente, se observa que:

Tabla No. 21

El docente cumple con los horarios de clases.

\begin{tabular}{|c|c|c|}
\hline & Frecuencia & Porcentaje \\
\hline Siempre & 629 & $79,3 \%$ \\
\hline Casi siempre & 96 & $12,1 \%$ \\
\hline A veces & 54 & $6,8 \%$ \\
\hline Casi nunca & 9 & $1,1 \%$ \\
\hline Nunca & 5 & $0,6 \%$ \\
\hline Total & 793 & $100,0 \%$ \\
\hline \multicolumn{2}{|c|}{ Fuente: Elaboración propia } \\
\end{tabular}

Tabla No. 22

Comparación del cumplimiento del docente con los horarios de clases según condición de reinscripción.

\begin{tabular}{|c|c|c|}
\hline & Con Reinscripción & Sin Reinscripción \\
\hline Siempre & $81,2 \%$ & $76,6 \%$ \\
\hline Casi siempre & $11,2 \%$ & $18,9 \%$ \\
\hline A veces & $5,7 \%$ & $3,6 \%$ \\
\hline Casi nunca & $1,3 \%$ & $0,0 \%$ \\
\hline Nunca & $0,6 \%$ & $0,9 \%$ \\
\hline Total & $100 \%$ & $100 \%$ \\
\hline
\end{tabular}

Fuente: Elaboración propia

De acuerdo con los datos analizados, respecto de la evaluación docente, no aparecen asociaciones entre las tres dimensiones analizadas (comprensión de explicaciones del docente, clima de trabajo y respeto por los horarios) y el abandono de los estudios.

Finalmente, combinando todas las variables mencionadas obtenemos el IRAD (Índice de Riesgo de Abandono Definitivo) que puede graficarse como sigue: 


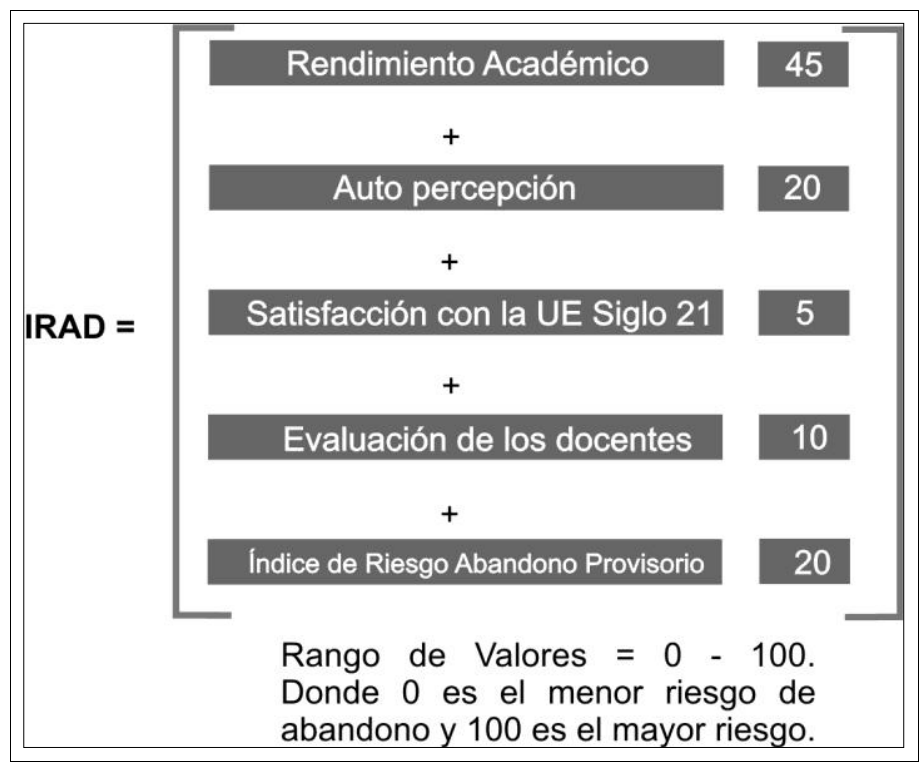

Figura No. 6. Índice de riesgo de abandono definitivo

Se observa en la figura 6 la ponderación (peso relativo) asignada a cada variable y el modo en el que se combinan. La misma surge de estudios exploratorios realizados en cohortes de estudiantes anteriores al año 2010 de la Universidad Siglo 21. Particularmente, se utilizó un modelo de regresión lineal binaria en la que el abandono fue incluido como variable dependiente, mientras que el resto de las variables (mencionadas en la figura 6) se incorporaron como independientes. Los resultados del modelo de regresión permitieron asignar el peso relativo de cada variable.

Los datos que se obtuvieron para el índice IRAD, en todos los estudiantes, son los siguientes:

Tabla No. 23

Índice de Riesgo de Abandono Definitivo (categorías equidistantes).

\begin{tabular}{|c|c|c|}
\hline & Frecuencia & Porcentaje \\
\hline Riesgo Muy Alto & 5 & $0,6 \%$ \\
\hline Riesgo Alto & 139 & $17,5 \%$ \\
\hline Riesgo Medio & 236 & $29,8 \%$ \\
\hline Riesgo Bajo & 253 & $31,9 \%$ \\
\hline Riesgo Muy Bajo & 160 & $20,2 \%$ \\
\hline Total & 793 & $100,0 \%$ \\
\hline
\end{tabular}

Fuente: Elaboración propia 
En la tabla 23 se observa que los mayores porcentajes del índice se concentran en los riesgos bajo y medio, aunque encontramos un $18,1 \%$ de estudiantes con riesgo alto y muy alto.

Finalmente, si comparamos los índices del IRAD con el abandono efectivo de los estudios, observaremos la siguiente relación:

Tabla No. 24

Comparación del Índice de Riesgo de Abandono Definitivo (categorías equidistantes) según condición de reinscripción.

\begin{tabular}{|c|c|c|}
\hline & Con Reinscripción & Sin Reinscripción \\
\hline Riesgo Muy Alto & $0,4 \%$ & $1,7 \%$ \\
\hline Riesgo Alto & $12,9 \%$ & $43,0 \%$ \\
\hline Riesgo Medio & $29,2 \%$ & $33,1 \%$ \\
\hline Riesgo Bajo & $34,4 \%$ & $18,2 \%$ \\
\hline Riesgo Muy Bajo & $23 \%$ & $4 \%$ \\
\hline Total & $100 \%$ & $100 \%$ \\
\hline
\end{tabular}

Fuente: Elaboración propia

En la tabla precedente puede observarse una muy interesante relación entre el abandono de los estudios y la predicción que establece el índice IRAD. Al respecto, notamos que entre aquellos alumnos que no se reinscribieron el $44,7 \%$ estaba previamente tipificado como con riesgo alto y muy alto, a diferencia del $13,3 \%$ en el caso de quienes no abandonaron sus estudios. $\mathrm{Si}$ a ello -tomando una posición conservadora- le sumáramos el riesgo medio obtendríamos que, entre aquellos que dejaron sus estudios, el $77,8 \%$ estaba tipificado con un riesgo medio a muy alto.

Cabe señalar que también encontramos alumnos que fueron calificados con riesgo bajo y muy bajo y, sin embargo, abandonaron sus estudios. Aún así, estos casos son significativamente menos que aquellos que presentaban riesgos medio a muy alto. 


\section{Discusión y reflexiones finales}

Los resultados de esta investigación constituyen solo el puntapié inicial de un proceso de trabajo que continúa indagando acerca de las variables relacionadas con el abandono de los estudios universitarios en el primer año de cursado. En esta línea, el modelo que hemos desarrollado -cuyos resultados son los índices IRAP e IRAD- ha sido aplicado por primera vez en el año 2010. Es decir, estos son los primeros resultados de una investigación en curso, por lo cual resulta imprescindible proseguir aplicando el modelo por varios años y analizando los datos que se obtengan.

Por el momento, si tuviésemos que dar respuesta a la pregunta de investigación que nos planteamos al comienzo de este artículo (respuesta que anticipamos con el supuesto/hipótesis inicial de trabajo), podríamos afirmar que la asociación entre las variables: A. Habilidades verbales, B. Grado de responsabilidad, C. Rendimiento académico, D. Autopercepción como alumno, E. Satisfacción con la Universidad, F. Evaluación de los docentes, y el abandono de los estudios en el primer año de cursado muestra resultados concluyentes en una de ellas, que es el rendimiento académico. Esta variable aparece como la más fuertemente asociada con el abandono. Sin embargo, la variable razonamiento verbal, correspondiente al test DAT, también muestra una incipiente relación con el abandono.

Las demás variables, por separado, no muestran -en esta primera experiencia de medición- asociaciones considerables con la variable abandono.

Cuando combinamos las variables mencionadas para conformar los dos índices IRAP e IRAD, vemos que el índice IRAP fue capaz de predecir el $22 \%$ de los alumnos que abandonaron sus estudios. Esto sólo si tomamos los puntajes de riesgo provisorio nominados como muy alto y alto. Si a ello le sumamos el puntaje medio, la predicción del índice trepa al $74 \%$.

Con el IRAD, sucede algo similar, aunque muestra mayor capacidad y precisión en la predicción. Así, el 44,7\% de los alumnos que abandonaron sus estudios estaba previamente tipificado como con un riesgo alto y muy alto. Si a ello -asumiendo una posición conservadorale sumáramos el riesgo medio, obtendríamos que, entre aquellos que dejaron sus estudios, el $77,8 \%$ estaba tipificado con un riesgo medio a muy alto.

Consideramos entonces que el desarrollo de estos dos índices muestra las siguientes aplicaciones: 
- El IRAP es obtenido antes de que los alumnos comiencen a cursar, por lo cual se transforma en un muy buen elemento para trabajar, inicialmente, sobre los alumnos que presentan riesgo muy alto y alto. $\mathrm{Y}$, dependiendo de los criterios pedagógicos $\mathrm{e}$ institucionales, también se podría trabajar con los alumnos que presentan riesgo medio. Ello, como lo hemos mencionado, sería una posición conservadora, pero quizá productiva para evitar el abandono temprano. En estos casos, el eje del trabajo pedagógico, basándose en los resultados del IRAP, debiera concentrarse en mejorar las capacidades de razonamiento verbal de los alumnos, dado que esta es la variable de IRAP que aparece más asociada con el abandono.

- En el caso del IRAD, su poder de predicción es mayor, pero hay que esperar al fin del ciclo (primer semestre) para obtener este índice. En algunas ocasiones, esto puede significar que ya es tarde para ciertos alumnos que abandonan antes de que termine el primer semestre. Sin embargo, encontramos posible señalar que podría trabajarse preventivamente con aquellos alumnos que muestran bajos resultados en sus primeras evaluaciones (evaluaciones parciales de sus materias). Esto constituye el comienzo de un bajo rendimiento académico y, como hemos observado, el rendimiento académico es la variable constitutiva de IRAD que más asociada está a la deserción.

Finalmente, a modo de discusión de aquí en adelante, se plantea la posibilidad de continuar con la investigación en la que se consideren periodos de tiempo más prolongados para el análisis de la deserción estudiantil. Resultaría interesante tomar la misma cohorte de alumnos, con la cual se trabajó sobre los índices IRAP e IRAD, y seguir monitoreando la condición de reinscripción a medida que avanza en sus estudios. Esto podría confirmar comportamientos (una vez que se producen las deserciones) o dar marcha atrás sobre las hipótesis de trabajo (como los que se reinscriben luego de haber abandonado).

\section{Referencias}

Ajzen, Icek y Fishbein, Martin. (1975). Attitudes toward objects as predictors of simple and multiple behavioural criteria. Psycological Review, 81, 59-74.

Bean, John. (1980). Dropouts and turnover: The synthesis and test of a causal model of student attrition. Research in Higher Education, 12 (4), 155-187. 
Bean John y Eaton, Shevawn Bogdan (2003). The psychology underlying successful retention practices. Journal of College Student Retention, 3 (1), 73-89.

Bennett, George, Seashore, Harold, y Wesman, Alexander. (1997). Test de aptitudes diferenciales DAT. Manual, Forma T. Buenos Aires: Paidós.

Breva Asensio, Alicia \& Amparo Carpi Ballester. (2002). La predicción de la conducta a través de los constructos que integran la teoría de la Acción Planeada. Revista Electrónica de

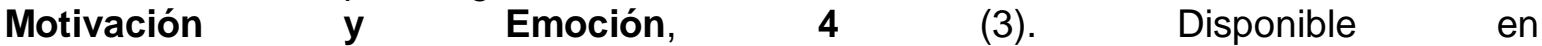
http://reme.uji.es/articulos/abreva7191302101/texto.html

Chalabe, Teresa, Pérez, Lilia y Truninger, Elizabeth. (2004). Reflexiones Sobre La Deserción Universitaria. Consultado el 11 de marzo de 2011 de http://rapes.unsl.edu.ar/Congresos realizados/Congresos/IV\%20Encuentro\%20-\%20Oct2004/eje7/14.htm

Costa, Paul y McCrae, Robert. (1999). Inventario de Personalidad NEO Revisado (NEO-PI-R) e Inventario NEO Reducido de Cinco Factores (NEO-FFI): Manual profesional. Madrid: TEA.

Díaz Peralta, Christian. (2008). Modelo conceptual para la deserción estudiantil universitaria chilena. Estudios Pedagógicos XXXIV (2). Valdivia.

Edel Navarro, Rubén. (2003). El rendimiento académico: concepto, investigación y desarrollo. REICE. Revista Electrónica Iberoamericana sobre calidad, eficacia y cambio en educación, 1 (2), Consultado el día 15 de Octubre de 2010 en: www.rieoei.org/investigacion/512Edel.PDF

Himmel, Erika. (2002). Modelos de análisis de la deserción estudiantil en la educación superior. Revista Calidad de la Educación, 17, 91-108.

Hyun-Joo Kim. (2003). Predicting an Individual Retention Rate Using Logistic Regression Analysis. Kirksville: Truman State University.

Ishiyama, John. (2000). The factors affecting retention, graduation and satisfaction rates at Truman State University: an initial empirical inquiry. Kirksville: Truman State University.

Morris, Jason. (2002). Academic integration, social integration, goal and institutional commitment. And spiritual integration. As predictors of persistence at a christian institution of higher education. Dissertation in higher education. Graduate Faculty of Texas Tech University (Mimeo). Disponible: http://thinktech.lib.ttu.edu/bitstream/handle/2346/16539/31295017082685.pdf?sequence=1 Consultado Marzo 2011

Reyes Rodríguez, Luis. (2007). La Teoría de la Acción Razonada. Implicaciones para el estudio de las actitudes. Investigación Educativa Duranguense, (7), 66-77. 
Rienks, Jane \& Stephanie Taylor. (2009). Attrition and academic performance of students identified as at-risk using administrative data alone. First year in Higher education Conference 2009. Brisbane: Queensland University of Technology

Spady, William. (1970). Dropouts from higher education: an interdisciplinary review and synthesis. Interchange, 19 (1), 109-121.

Tinto, Vincent. (2008). Definir la deserción: una cuestión de perspectiva. Consultado el día 2 de Marzo de 2011 en: http://matematicas.reduaz.mx/mat01/DES/k/deser.pdf

Tinto, Vincent. (1997) Classrooms as Communities: Exploring the Educational Character of Student Persistence. Journal of Higher Education, 68 (6).

Tinto, Vincent. (1992). El abandono de los estudios superiores: una nueva perspectiva de las causas del abandono y su tratamiento. México: ANUIES.

Tinto, Vincent. (1982). Limits of theory and practice of student attrition. Journal of Higher Education, 53 (6), 87-100.

Tinto, Vincent. (1975). Dropout in higher education: A theoretical synthesis of recent research. Review of Educational Research, 45 (1), 89-125. 